\title{
BUFET: boosting the unbiased miRNA functional enrichment analysis using bitsets
}

\author{
Konstantinos Zagganas ${ }^{1,2^{*}}$ (D), Thanasis Vergoulis², Maria D. Paraskevopoulou ${ }^{3,4}$, loannis S. Vlachos ${ }^{3,4}$, \\ Spiros Skiadopoulos ${ }^{1}$ and Theodore Dalamagas ${ }^{2}$
}

\begin{abstract}
Background: A group of miRNAs can regulate a biological process by targeting genes involved in the process. The unbiased miRNA functional enrichment analysis is the most precise in silico approach to predict the biological processes that may be regulated by a given miRNA group. However, it is computationally intensive and significantly more expensive than its alternatives.

Results: We introduce BUFET, a new approach to significantly reduce the time required for the execution of the unbiased miRNA functional enrichment analysis. It derives its strength from the utilization of efficient bitset-based methods and parallel computation techniques.
\end{abstract}

Conclusions: BUFET outperforms the state-of-the-art implementation, in regard to computational efficiency, in all scenarios (both single- and multi-core), being, in some cases, more than one order of magnitude faster.

Keywords: miRNAs, Functional enrichment analysis, BUFET

\section{Background}

microRNAs (miRNAs) are short ( 23nt) non-coding RNA molecules that are considered to be central gene expression regulators. They act through mRNA degradation and/or translational suppression of protein coding transcripts. By binding to specific recognition elements with perfect or imperfect base complementarity, miRNAs interact with genes and inhibit their expression. Consequently, they can play a key role in the regulation of numerous biological processes and, thus, miRNA-induced up- or down-regulation can be indicative of a diseased state [1-3]. On the other hand, each miRNA can target hundreds of different genes [4] and its perturbed expression can, in turn, affect numerous biological functions. This makes the analysis of the effects of miRNAs on biological processes crucial to the understanding of this post-transcriptional regulation mechanism.

miRNA functional enrichment analysis is the in silico process which enables researchers to discover potential

\footnotetext{
*Correspondence: kzagganas@uop.gr

${ }^{1}$ University of Peloponnese, Department of Informatics and

Telecommunications, 22100 Tripoli, Greece

2"Athena" Research and Innovation Center, 15125 Athens, Greece

Full list of author information is available at the end of the article
}

biological functions affected by a group of differentially expressed miRNAs. The first step of this process is the identification of all genes targeted by at least one of the miRNAs in the group. In most cases, these gene sets are produced by target prediction algorithms like DIANAmicroT [5, 6], miRanda [7] or TargetScan [8]. Then, gene annotation data (e.g., pathways, functions, etc) for all known genes are collected. These data are usually retrieved by the Gene Ontology (GO) Consortium [9] (or other sources like KEGG [10, 11] and PANTHER [12]) and capture the involvement of genes in several biological processes. Finally, a statistical analysis is applied on the data collected during the previous two steps, to reveal the annotation categories that are overrepresented in the genes targeted by the miRNA group. Usually, the algorithm selected for this step is Fisher's exact test [13, 14], which calculates $p$-values based on the hypergeometric distribution.

However, it has recently been shown [15] that the use of the aforementioned statistics approach can produce significant $p$-values even for biological processes, controlled by groups of randomly selected miRNAs. This indicates that an underlying bias exists between miRNAs, their predicted gene-targets and the structure of the 
annotation, also reflected in the performed enrichment analyses. Thus, in order to overcome this problem, the authors of [15] proposed a Monte Carlo test which produces an empirical p-value. Moreover, as pointed out by the authors of [16], this approach moves the analysis from the gene to the miRNA level by defining the biological process overlap as the proportion of those genes that are both targeted by the miRNA group of interest and also involved in the biological process under examination. In brief, their approach is the following: first, a large number of randomly assembled miRNA groups having the same number of miRNAs as the group of interest are selected. Then, the empirical $p$-value is defined as the proportion of those random groups that exhibit a greater biological process overlap than the miRNA group under examination. More details on the benefits of this approach to miRNA functional enrichment analysis are available in the original paper [15] by Bleazard et al.

The number of random miRNA groups selected to perform the analysis is a parameter that controls the accuracy of the $p$-value to be produced. In particular, the higher the number of random miRNA groups selected, the more accurate the produced $p$-value will be. Usually, 1 million random groups are used to achieve sufficient accuracy [15]. Unfortunately, using such a large number of groups results in unreasonably large execution times. For example, an execution of the state-of-the-art implementation [15] for a group of 100 miRNAs as input, using 1 million random groups, on a single core of an Intel i7-3820 processor requires up to $17 \mathrm{~h}$ of processing time.

In order to alleviate this issue, we introduce BUFET (Bitset-based Unbiased miRNA Functional Enrichment Tool). This approach exploits efficient data structures to significantly reduce the execution time of the unbiased enrichment analysis. BUFET also takes advantage of parallel computing techniques to achieve additional performance improvements in multi-core systems. The contribution of this work can be summarized in the following:

- We studied the computational requirements and examined the performance bottlenecks of the unbiased miRNA functional enrichment analysis.

- We investigated the performance of different data structures, namely hash tables and bitsets, in regards to their effectiveness in unblocking the identified bottlenecks.

- We developed BUFET, a tool that utilises the results of the aforementioned investigation to boost the speed of the unbiased miRNA functional enrichment analysis. To achieve an even greater speed boost in the case of multi-core environments, we exploited multithreading to implement parallel execution of the analysis.
- We performed an extensive evaluation of BUFET to demonstrate its efficiency. BUFET outperforms the state-of-the-art approach in all scenarios (in many cases by an order of magnitude).

- We provide BUFET as an open source implementation, which is freely available on GitHub (see the "Availability and requirements" section). BUFET is a powerful tool that provides flexible input file formats enabling many execution modes (e.g., execution using custom miRNA-gene interactions and gene annotations).

\section{Implementation}

\section{The challenge}

As mentioned previously, the unbiased miRNA functional enrichment analysis involves the examination of a large number of biological processes (or, equivalently, annotation categories) to identify those, which are more likely to be affected by the gene-targets of a miRNA group. During this type of analysis, both biological processes and miRNAs are represented as gene sets: each biological process is represented by the genes involved in it, while each miRNA by its gene-targets.

It becomes evident that computing the biological process overlap of a miRNA group (see the "Background" section) involves the calculation of the intersection between the set of genes targeted by the miRNA group and the set of genes involved in the biological process. Moreover, the set of genes targeted by each miRNA group needs to be calculated "on the fly" by performing union operations on the gene sets of each miRNA in the group. Therefore, the unbiased miRNA functional enrichment analysis relies on performing a very large number of set unions and intersections. For instance, for a given query miRNA group of size 10, about 10 million unions and more than 8 billion intersections are required to produce a $p$-value.

The state-of-the-art implementation of the unbiased miRNA functional enrichment analysis [15] uses hash tables (more specifically, Python sets ${ }^{1}$ ) to represent gene sets. The advantage of this data structure is that performing union and intersection operations for small sets is usually very fast. Both operations are performed by executing a variant of the hash-join algorithm [17]. On the other hand, hash-join becomes very inefficient when operating on large sets.

Unfortunately, in the case of the unbiased miRNA functional enrichment analysis, all union operations are performed on large gene sets. This is attributed to the fact that each of these gene sets corresponds to the predicted targets of a particular miRNA. Since miRNA target prediction algorithms usually produce hundreds or even thousands of results (interactions) for a single miRNA, 
it becomes evident that most of the performed union operations can be quite slow if hash-join is used.

To overcome this problem, the bitset (or bit-vector) [17], an alternative data structure, which is more suitable for the representation of large sets, can be used. When sets of genes are implemented as bitsets, unions and intersections between them can be calculated by performing bitwise operations on bit blocks. In particular, bitwise-or can be used to get the union of two sets, while bitwi se-and to get their intersection. Such operations are efficient for large sets, since their execution time is not affected by the size of the set ${ }^{2}$. Additionally, the representation of gene sets as bitsets is more efficient, memory-wise, in the case of relatively large sets of genes (like those produced by miRNA target prediction algorithms).

The calculation of the targets of each miRNA group would benefit greatly by the use of bitwise-or, since, as previously mentioned, it involves a large number of union operations on large gene sets represented by dense bitsets. In this case, bitsets also have a reduced memory footprint compared to hash-tables. On the other hand, gene sets related to biological processes, as provided by Gene Ontology annotations [9], usually consist of a small number of genes. Therefore, hash-join on these sets can be rather efficient ${ }^{3}$.

The previous discussion suggests that a hybrid solution, using bitwise operations for unions and hash-join for intersections, seems more suitable than both of the aforementioned approaches. Unfortunately, this hybrid approach has a major drawback. The gene sets generated by bitwise-or for all miRNA groups must be provided as input to the hash-join algorithm for the calculation of the biological process overlaps. However, the bitwise-or algorithm produces gene sets represented as bitsets, while hash-join requires its input in the form of hash tables. Therefore, a data structure conversion must be performed, introducing an important execution overhead that counterbalances any gains in efficiency.

\section{The BUFET approach}

Our approach, called BUFET, is demonstrated in Fig. 1. It combines the best characteristics of bitset- and hash-table-based methods without suffering from the aforementioned shortcomings of a hybrid approach. It takes advantage of the efficiency of bitwise-or in calculating the union of large sets to produce the gene sets targeted by particular miRNA groups. These gene sets are represented as bitsets, called miRNA group bitsets.

Meanwhile, the biological process overlap of each miRNA group is calculated as follows: for each gene annotated as part of the biological process, the respective bit in the miRNA group bitset is examined. If the bit is set, then the value of a counter is increased by one (its value is initially zero). Otherwise, the value of the counter remains intact. After all genes related to the biological process have been considered, the value of the counter provides the size of the intersection and, subsequently, the biological process overlap. Since the genes are used to probe the miRNA group bitset, we refer to this method as bit-probing.

Further optimizations were introduced in order to achieve additional performance improvements. First, biological processes that have no common genes with the miRNA group under examination can be excluded from the analysis (since no interference by the miRNAs in the group with the process is recorded). Additionally, BUFET supports full utilization of multi-core computing systems by supporting parallelization at the biological process level.

It should be noted that parallel execution is also supported by the state-of-the-art approach presented in [15]. However, in contrast to the use of multiprocessing adopted by this approach to implement parallelization,

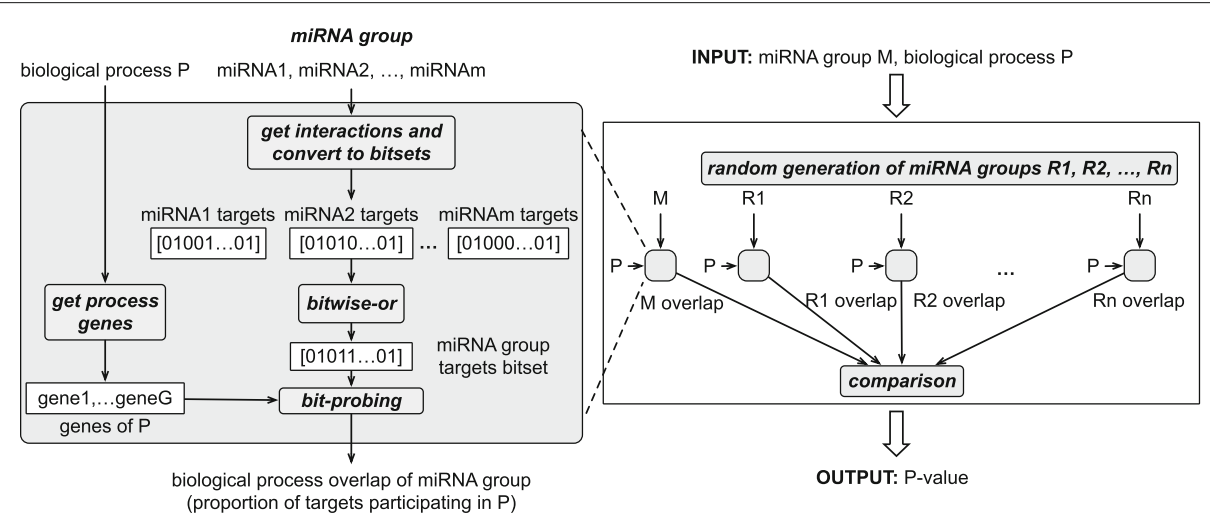

Fig. 1 Flowchart summarizing the BUFET approach 
BUFET uses multithreading. The advantage of multithreading over multiprocessing is that all processes running in parallel have access to the same part of the main memory. This eliminates the need to copy data across processes, thus reducing the execution time and memory footprint.

On the other hand, an issue with this approach is that the bitsets containing the targets of the random miRNA groups have to be calculated and stored in main memory. This step is necessary, so that every thread is able to access the data in order to calculate a $p$-value. Consequently, this increases the memory footprint, although, the amount of memory required does not pose a big challenge for contemporary computers. More specifically, none of the many real-world analysis scenarios examined during our experiments resulted in the allocation of more than $3.5 \mathrm{~GB}$ of RAM to our script.
Table 1 Statistics related to the miRNA-to-gene interactions used

\begin{tabular}{lllllll}
\hline & \multicolumn{4}{c}{ Number of genes/miRNA } & Total miRNAs \\
\cline { 3 - 6 } & \multicolumn{4}{c}{ Minimum Maximum Average } & & \multicolumn{3}{c}{ Median Std. } \\
& \multicolumn{4}{c}{ Deviation } \\
\hline microT & 1 & 4547 & 404 & 206 & 459 & 2580 \\
miRanda & 11 & 6977 & 1309 & 1096 & 932 & 2588 \\
\hline
\end{tabular}

\section{Functionality and source code}

BUFET is provided as a free, open source software licensed under GPL v3 (a download link is provided in the "Availability and requirements" section). Its core is implemented in $\mathrm{C}++$ for greater efficiency, while a Python wrapper script facilitates its execution and its incorporation in existing bioinformatics workflows.

The input of the BUFET software consists mainly of two CSV files: one containing miRNA-to-gene interactions

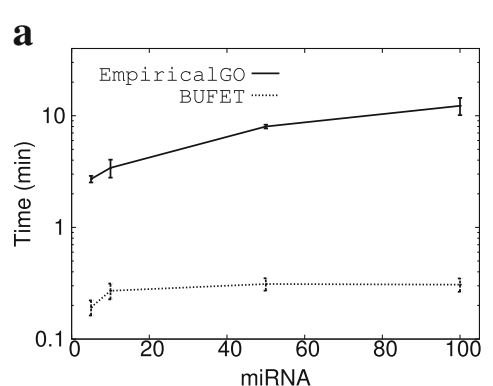

microT, 10K random groups

c

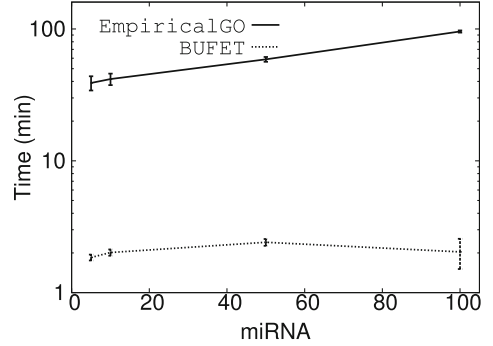

microT, $100 \mathrm{~K}$ random groups

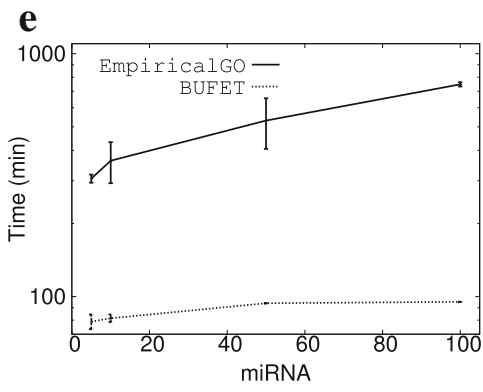

microT, $1 \mathrm{M}$ random groups

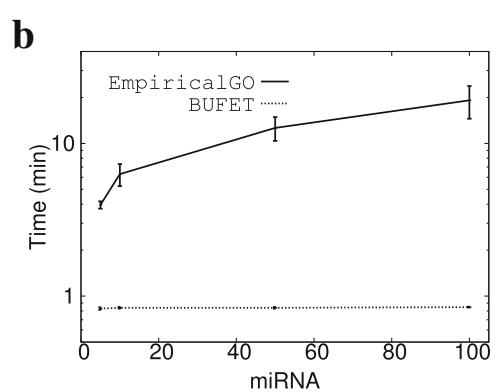

miRanda, 10K random groups

d

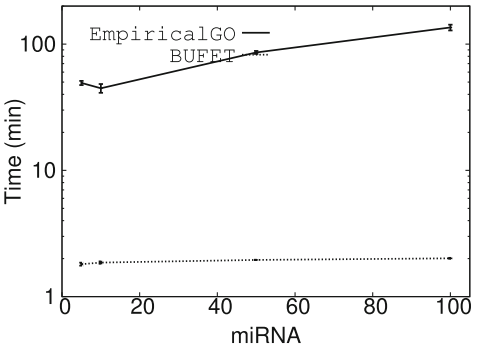

miRanda, 100K random groups

f

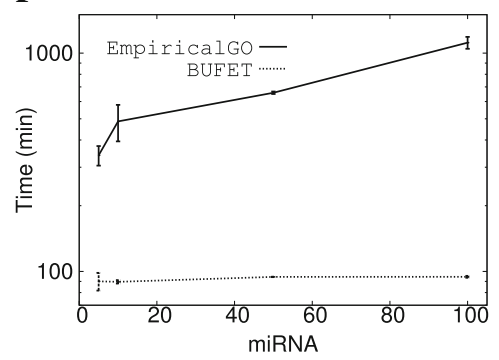

miRanda, $1 \mathrm{M}$ random groups

Fig. 2 Average execution times (log scale) on a single core with a varying number of miRNAs. (a) microT, 10K random groups. (b) miRanda, 10K random groups. (c) microT, 100K random groups. (d) miRanda, 100 Krandom groups. (e) microT, 1M random groups. (f) miRanda, $1 \mathrm{M}$ random groups 
and another containing associations of biological functions with particular genes. The proper format of these files is described in the software download page. It should be noted that BUFET provides flexibility, enabling the users to upload miRNA-to-gene interactions based on the prediction algorithm of their choice (e.g., TargetScan [8], DIANA-microT [5, 6], miRanda [7], etc.) and to use biological function annotations collected by their preferred source (e.g., GO [9], KEGG [10, 11], or PANTHER [12]).

Finally, BUFET also performs Benjamini-Hochberg FDR correction [18]. More specifically, following the method in [19], we assume that $5 \%$ (and 1\%) of the produced $p$ values (under the 0.05 threshold) are false positives, while the rest are significant results. $P$-values significant at FDR 0.05 are marked with "*:" while $p$-values significant at 0.01 are marked with “*:*" in the output file.

\section{Results}

In this section, the efficiency of BUFET is evaluated against that of the state-of-the-art implementation (EmpiricalGO ${ }^{4}$, in both single- and multi-core environments. First, we examine the effect of the miRNA group size on the execution times of both implementations. Next, we investigate their parallel behavior for a varying number of CPU cores. miRNA-to-gene interactions were collected from DIANA-microT-CDS (score threshold $=0.8$ ) and miRanda (score threshold $=155$ and free energy $=-20$ ), while GO annotation data were obtained from Ensembl. Statistics related to miRNA-togene-interactions data used are presented in Table 1. All experiments were executed on a machine powered by an Intel Core i7-3820 processor with 8 cores (4 physical) and $64 \mathrm{~GB}$ of main memory.

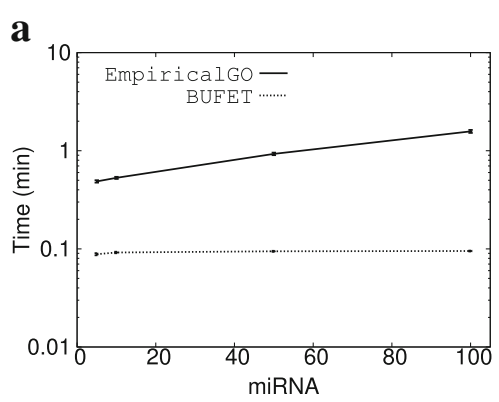

microT, $10 \mathrm{~K}$ random groups

c

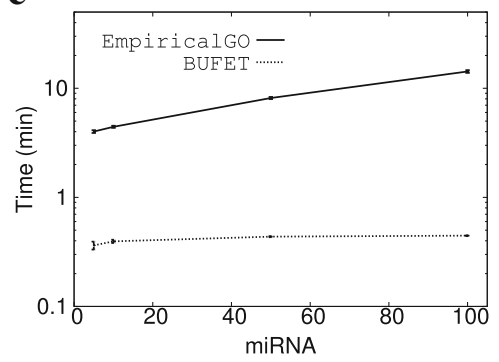

microT, $100 \mathrm{~K}$ random groups

e

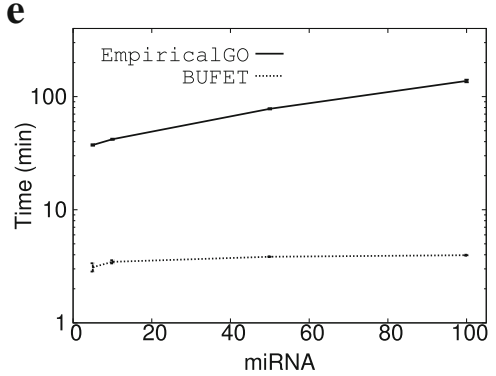

microT, $1 \mathrm{M}$ random groups b

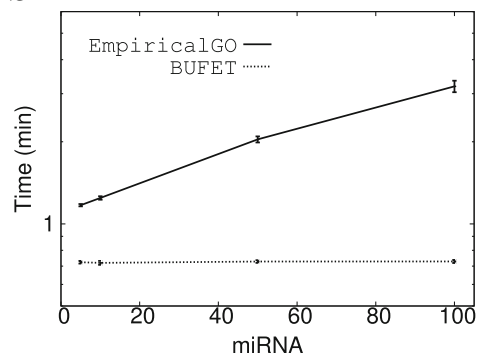

miRanda, 10K random groups

d

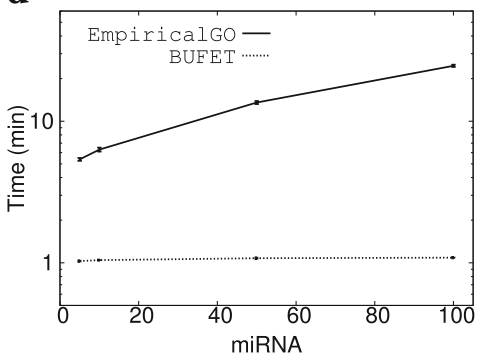

miRanda, $100 \mathrm{~K}$ random groups

f

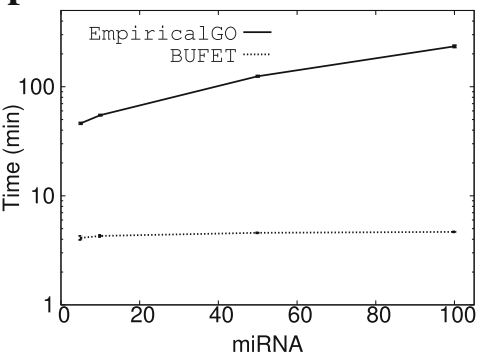

miRanda, $1 \mathrm{M}$ random groups

Fig. 3 Average execution times (log scale) on 7 cores with a varying number of miRNAs. a microT, 10K random groups. b miRanda, 10K random groups. c microT, $100 \mathrm{~K}$ random groups. d miRanda, $100 \mathrm{~K}$ random groups. e microT, $1 \mathrm{M}$ random groups. $\mathbf{f}$ miRanda, $1 \mathrm{M}$ random groups 


\section{Varying the miRNA group size}

Figure 2 presents (a) the average execution time of BUFET and EmpiricalGO and (b) its standard deviation (using error bars) for each measurement point and for varying miRNA group sizes (5, 10, 50 and 100 miRNAs) in a single-core environment. For each miRNA group size, 10 different groups were used as input to both implementations. Thus, every reported execution time is the average of 10 executions. The left column corresponds to the experiment performed using DIANA-microT-CDS interactions, while the right to the one using miRanda interactions. We performed each experiment by selecting the following, commonly-used settings: 10 thousand (10K), 100 thousand (100K), and 1 million (1M) random miRNA groups. Since the difference in the execution times between EmpiricalGO and BUFET are very large, all diagrams are presented in log scale for the y axis to enhance legibility.

It is clear that the execution time increases as the number of miRNAs in the group under examination increases for both approaches (due to the larger number of union operations that have to be performed). However, it is evident that the rate of the increase in the execution time is larger for EmpiricalGO than BUFET. This can be attributed to the fact that BUFET exploits the efficiency of bitwise-or in calculating unions on large gene sets. It also becomes evident that BUFET scales better than EmpiricalGO and in some cases, it is faster by at least an order of magnitude. Therefore, BUFET is a very efficient approach when high accuracy is needed for functional analysis of large miRNA groups.

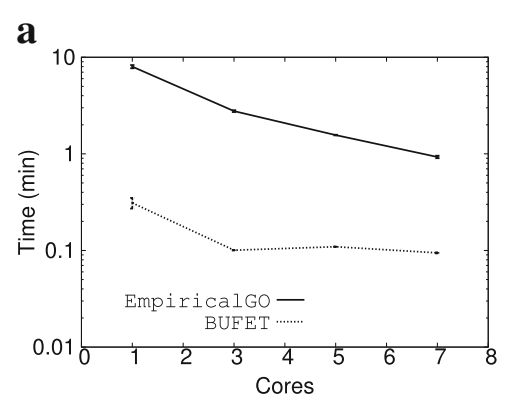

microT, $10 \mathrm{~K}$ random groups

c

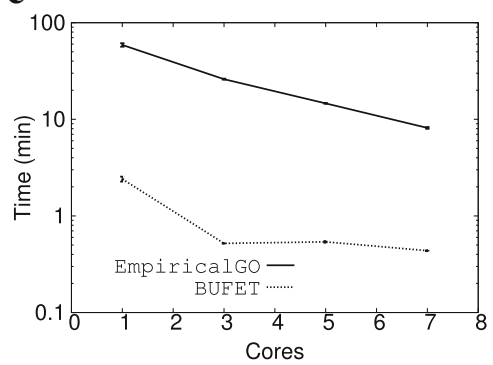

microT, $100 \mathrm{~K}$ random groups

e

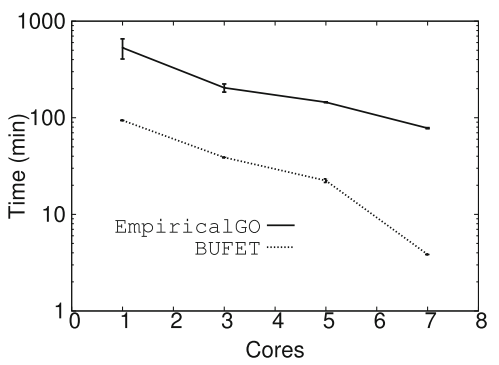

microT, $1 \mathrm{M}$ random groups b

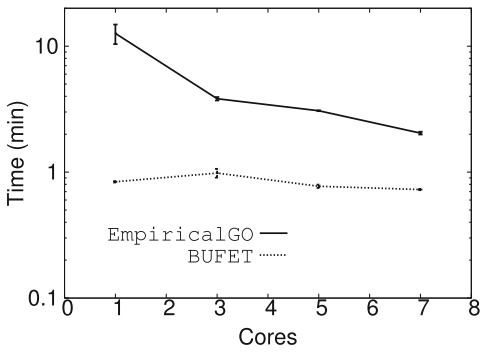

d

miRanda, 10K random groups

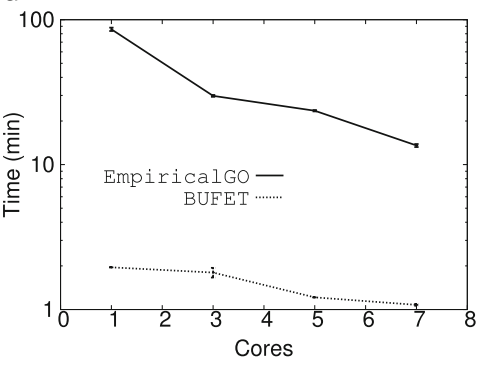

miRanda, 100K random groups

f

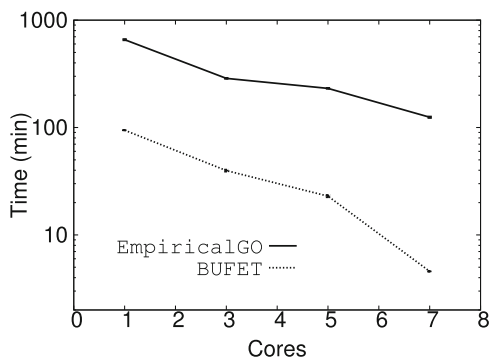

miRanda, 1M random groups

Fig. 4 Average execution times (log scale) varying the number of cores. a microT, 10K random groups. b miRanda, 10K random groups. c microT, $100 \mathrm{~K}$ random groups. $\mathbf{d}$ miRanda, $100 \mathrm{~K}$ random groups. e microT, $1 \mathrm{M}$ random groups. $\mathbf{f}$ miRanda, $1 \mathrm{M}$ random groups 
Figure 3 shows the same experiments in a multi-core environment ( 7 cores were used). Note that the main trends observed in the single-core experiment continue to occur: increasing the miRNA group size leads to increased execution times for both methods, while BUFET is significantly more efficient than EmpiricalGo in all cases. Note that, for the case of 5 miRNAs in low accuracy mode, the execution times tend to converge to the time needed for serial operations (i.e. file reading, output writing, and FDR correction). Finally, it is worth mentioning that, in the case of 100 miRNAs using 7 cores, in high accuracy mode, BUFET can produce results in under 5 min, while EmpiricalGo needs more than $7 \mathrm{~h}$ for the samel task.

\section{Varying the number of cores}

Figure 4 shows the average time required by BUFET and EmpiricalGo to calculate the empirical $p$-values for 10 input groups of size 50 by using a varying number of $C P U$ cores. It is clear that both approaches become faster as the number of cores increases. However, in every case BUFET requires significantly less time to execute.

\section{Conclusion}

In this paper we dealt with the performance of the unbiased miRNA functional enrichment analysis. We showed that the state-of-the-art approach to perform this type of analysis (EmpiricalGO) is not practical in terms of computational efficiency, especially for large miRNA groups when high accuracy is required. To deal with this problem we introduced BUFET, an alternative bitset-based approach. Our experiments make evident that BUFET outperforms the state-of-the-art implementation in all scenarios (in many cases by orders of magnitude). Additionally, the better scalability of BUFET makes it a very appealing solution for the analysis of large miRNA groups when 1 million random groups are used for the analysis. Note that, BUFET is provided as an open source implementation which is freely available on GitHub (the download URL is provided in the "Availability and requirements" section).

\section{Availability and requirements \\ Project name: BUFET}

Project home page: https://github.com/diwis/BUFET/

Operating system(s): Linux, MacOSX.

Programming language: $\mathrm{C}++$, Python.

Other requirements: Python interpreter 2.7 or higher, $\mathrm{g}++4.8$ or higher.

License: GNU GPL v.3.

Any restrictions to use by non-academics: None

\section{Endnotes}

${ }^{1}$ https://docs.python.org/3/tutorial/datastructures. html
${ }^{2}$ In particular, the execution time of each bitwise operation depends on the number of bits it contains, i.e., on the cardinality of the set's domain.

${ }^{3}$ Regarding the calculation of the biological process overlap, an additional optimization is possible for the hash-join algorithm. In particular, the production of the output intersection set can be avoided, since only its size is required. However, a similar optimization is not feasible for bitwise-and.

${ }^{4}$ http://sgjlab.org/empirical-go/

\section{Abbreviations}

BUFET: Bitset-based unbiased functional enrichment tool; CPU: Central processing unit; CSV: Comma-separated values; FDR: False discovery rate; GB: Gigabytes; GO: Gene ontology; GPL: General public licence; KEGG: Kyoto encyclopedia of genes and genomes; miRNAs: microRNAs; PANTHER: Protein ANalysis THrough evolutionary relationships; RAM: Random access memory; URL: Uniform resource locator

\section{Acknowledgements}

We would like to thank Prof. Artemis G. Hatzigeorgiou for her scientific and technical assistance to our work as well as her valuable comments towards improving the manuscript.

\section{Funding}

This work was funded by the European Commission under the Research Infrastructure (H2020) programme (project: ELIXIR-EXCELERATE, grant: GA676559).

\section{Availability of data and materials}

The software, together with instructions for use, can be found at https:// github.com/diwis/BUFET/.

The data sets generated and/or analysed during the current study are available in the following repositories:

- microT miRNA-gene interactions for miRBase v.21 and Ensembl v.69: available at http://diana.imis.athena-innovation.gr/DianaTools/index. php?r=microT_CDS/index. Experiment dataset available at: http:// carolina.imis.athena-innovation.gr/bufet/microT_dataset.csv

- miRanda miRNA-gene interactions for miRBase v.21 and Ensembl v.69: created by running the miRanda software (http://www.microrna.org/ microrna/getDownloads.do. Experiment dataset available at: http:// carolina.imis.athena-innovation.gr/bufet/miRanda_dataset.csv.

- GO gene annotations for Ensembl v.69. Experiment dataset available at: http://carolina.imis.athena-innovation.gr/bufet/annotation_dataset.csv

- Input for the experiments presented in the paper is available in a zip file at: http://carolina.imis.athena-innovation.gr/bufet/experiment_input.zip.

\section{Reproducibility of experiments}

All experiments presented in this paper are reproducible using the input and interaction files provided in the "Availability of data and material". Code for BUFET and EmpiricalGO as well as instructions for execution were retrieved by the links provided in the "Availability and requirements" section and footnote 4.

\section{Authors' contributions}

MP and IV provided the motivation for BUFET. KZ, TV, and TD designed the algorithm. KZ implemented the algorithm, built the experimental platform, and performed the experiments. MP, IV, and SS provided useful insight to interpret the experimental results. TV and TD provided guidance on the implementation and the experiments. All authors read, contributed to and approved the final manuscript.

Ethics approval and consent to participate Not applicable. 


\section{Consent for publication}

Not applicable.

\section{Competing interests}

The authors declare that they have no competing interests.

\section{Publisher's Note}

Springer Nature remains neutral with regard to jurisdictional claims in published maps and institutional affiliations.

\section{Author details}

${ }^{1}$ University of Peloponnese, Department of Informatics and

Telecommunications, 22100 Tripoli, Greece. " "Athena" Research and Innovation Center, 15125 Athens, Greece. ${ }^{3}$ DIANA-Lab, Department of Electrical \& Computer Engineering, University of Thessaly, 38221 Volos, Greece.

${ }^{4}$ Hellenic Pasteur Institute, 127 Vasilissis Sofias Avenue, 11521 Athens, Greece.

Received: 17 January 2017 Accepted: 29 August 2017

Published online: 06 September 2017

\section{References}

1. Leidinger P, Backes C, Deutscher S, Schmitt K, Mueller SC, Frese K, Haas J, Ruprecht K, Paul F, Stahler C, Lang CJ, Meder B, Bartfai T, Meese $\mathrm{E}$, Keller A. A blood based 12-miRNA signature of Alzheimer disease patients. Genome Biol. 2013;14(7):78.

2. Schratt GM, Tuebing F, Nigh EA, Kane CG, Sabatini ME, Kiebler M, Greenberg ME. A brain-specific microrna regulates dendritic spine development. Nature. 2006;439(7074):283-9.

3. Xie F, Wang Q, Sun R, Zhang B. Deep sequencing reveals important roles of micrornas in response to drought and salinity stress in cotton. J Exp Bot. 2015;66(3):789-804. doi:10.1093/jxb/eru437. http://jxb.oxfordjournals.org/content/66/3/789.full.pdf+html.

4. Friedman RC, Farh $\mathrm{KK}-\mathrm{H}$, Burge CB, Bartel DP. Most mammalian mrnas are conserved targets of micrornas. Genome Res. 2009;19(1):92-105.

5. Paraskevopoulou MD, Georgakilas G, Kostoulas N, Vlachos IS, Vergoulis T, Reczko M, Filippidis C, Dalamagas T, Hatzigeorgiou AG. Diana-microt web server v5.0: service integration into mirna functional analysis workflows. Nucleic Acids Res. 2013;41(W1):169-73. doi:10.1093/nar/gkt393. http://nar.oxfordjournals.org/content/41/W1/W169.full.pdf+html.

6. Reczko M, Maragkakis M, Alexiou P, Grosse I, Hatzigeorgiou AG. Functional microrna targets in protein coding sequences. Bioinformatics. 2012;28(6):771-6. doi:10.1093/bioinformatics/bts043. http://bioinformatics.oxfordjournals.org/content/28/6/771.full.pdf+html.

7. John B, Enright AJ, Aravin A, TuschI T, Sander C, Marks DS. Human microrna targets. PLoS Biol. 2004;2(11). doi:10.1371/journal.pbio.0020363.

8. Lewis BP, Burge CB, Bartel DP. Conserved seed pairing, often flanked by adenosines, indicates that thousands of human genes are microrna targets. Cell. 120(1):15-20. doi:10.1016/j.cell.2004.12.035.

9. Ashburner M, Ball CA, Blake JA, Botstein D, Butler H, Cherry JM, Davis AP, Dolinski K, Dwight SS, Eppig JT, Harris MA, Hill DP, Issel-Tarver L, Kasarskis A, Lewis S, Matese JC, Richardson JE, Ringwald M, Rubin GM, Sherlock G. Gene ontology: tool for the unification of biology. Nat Genet. 2000;25(1):25-9.

10. Kanehisa M, Sato Y, Kawashima M, Furumichi M, Tanabe M. Kegg as a reference resource for gene and protein annotation. Nucleic Acids Res. 2016;44(D1):457-62. doi:10.1093/nar/gkv1070. http://nar.oxfordjournals.org/content/44/D1/D457.full.pdf+html.

11. Kanehisa M, Goto S. Kegg: Kyoto encyclopedia of genes and genomes. Nucleic Acids Res. 2000;28(1):27-30. doi:10.1093/nar/28.1.27. http://nar.oxfordjournals.org/content/28/1/27.full.pdf+html.

12. Thomas PD, Campbell MJ, Kejariwal A, Mi H, Karlak B, Daverman R, Diemer K, Muruganujan A, Narechania A. Panther: a library of protein families and subfamilies indexed by function. Genome Res. 2003;13(9): 2129-41.

13. Fisher RA. Statistical methods for research workers In: Kotz S, Johnson NL, editors. Breakthroughs in Statistics: Methodology and Distribution. New York: Springer New York; 1992. p. 66-70.

14. Vlachos IS, Zagganas K, Paraskevopoulou MD, Georgakilas G, Karagkouni D, Vergoulis T, Dalamagas T, Hatzigeorgiou AG. Diana-mirpath v3.0: deciphering microrna function with experimental support. Nucleic Acids Res. 2015. doi:10.1093/nar/gkv403. http://nar. oxfordjournals.org/content/early/2015/05/14/nar.gkv403.full.pdf+html.

15. Bleazard T, Lamb JA, Griffiths-Jones S. Bias in microrna functional enrichment analysis. Bioinformatics. 2015. doi:10.1093/bioinformatics/ btv023. http://bioinformatics.oxfordjournals.org/content/early/2015/02/ 18/bioinformatics.btv023.full.pdf+html.

16. Garcia-Garcia F, Panadero J, Dopazo J, Montaner D. Integrated gene set analysis for microrna studies. Bioinformatics. 2016;32(18):2809-16. doi:10.1093/bioinformatics/btw334. http://bioinformatics.oxfordjournals.org/content/32/18/2809.full.pdf+html.

17. Frakes WB, Baeza-Yates $\mathrm{R}$, (eds). Information retrieval: data structures and algorithms. Upper Saddle River: Prentice-Hall, Inc.; 1992.

18. Yoav Benjamini YH. Controlling the false discovery rate: A practical and powerful approach to multiple testing. J R Stat Soc Ser B Methodol. 1995;57(1):289-300.

19. McDonald JH. Multiple comparisons: Controlling the false discovery rate: Benjamini-Hochberg Procedure. Baltimore: Sparky House Publishing; 2014, pp. 254-60. Handbook of Biological Statistics.

\section{Submit your next manuscript to BioMed Central and we will help you at every step:}

- We accept pre-submission inquiries

- Our selector tool helps you to find the most relevant journal

- We provide round the clock customer support

- Convenient online submission

- Thorough peer review

- Inclusion in PubMed and all major indexing services

- Maximum visibility for your research

Submit your manuscript at www.biomedcentral.com/submit
C Biomed Central 